\title{
Validation of the Short Obsessive-Compulsive Disorder Screener (SOCS) in children and adolescents
}

José A. Piqueras, Tíscar Rodríguez-Jiménez, Ana G. Ortiz, Elena Moreno, Luisa Lázaro and

Antonio Godoy

\section{Background}

The Short Obsessive-Compulsive Disorder Screener (SOCS) is recommended by the National Institute for Health and Care Excellence as a suitable and validated screening tool for 11- to 15-year olds. Despite its excellent sensitivity and specificity in detecting obsessive-compulsive disorder (OCD), it has limitations

\section{Aims}

To empirically examine whether the SOCS is suitable for assessing OCD symptoms across a wide age range of children and adolescents and to provide new data about its psychometric properties.

\section{Method}

Participants were 94 patients (9-19 years) with OCD, and 880 healthy controls.

\section{Results}

The results supported the SOCS' unidimensional factor structure and metric invariance across samples. It showed good reliability in terms of internal consistency and temporal stability. Furthermore, it had significantly high correlations with other OCD measures and an acceptable sensitivity and specificity for detecting OCD.

\section{Conclusions}

The SOCS is a brief screening tool suitable for detecting OCD in children and adolescents.

\section{Declaration of interest}

None.

\section{Copyright and usage}

(C) The Royal College of Psychiatrists 2015. This is an open access article distributed under the terms of the Creative Commons Non-Commercial, No Derivatives (CC BY-NC-ND) licence.
Obsessive-compulsive disorder (OCD) symptoms in children and adolescents are an underestimated and understudied problem. Recently, OCD has become part of a new chapter - entitled 'Obsessive compulsive and related disorders' - in the DSM-5. ${ }^{1}$ $\mathrm{OCD}$ is a mental disorder characterised by the presence of obsessions and/or compulsions that produce intense distress and disability. ${ }^{1}$ According to epidemiological studies, OCD often begins in childhood or early adolescence. ${ }^{2}$ Prevalence rates in children and adolescents can reach up to $3 \%$ for $\mathrm{OCD}^{3}$ and up to $19 \%$ for the subclinical symptoms. ${ }^{3-5}$

Therefore, the development and dissemination of effective interventions for OCD are critically important, as is the need for early detection and intervention to improve outcomes. For example, regarding assessment and treatment of OCD, the National Institute for Health and Care Excellence (NICE) recommends the routine screening of young people at risk in general practice or other settings where they may present for help. ${ }^{6}$ Such screening requires short, easy-to-use, highly sensitive, reasonable and widely available measures. ${ }^{7}$

Thus, recently, there has been a considerable increase in assessment tools for OCD and obsessive-compulsive symptoms in children and adolescents. ${ }^{8}$ For example, according to a recent review, ${ }^{9}$ the Children's Yale-Brown Obsessive-Compulsive Scale $(\mathrm{CY}-\mathrm{BOCS})^{10}$ is the gold standard in the field of psychiatric clinical assessment, because of its excellent psychometric properties; however, it is a lengthy, clinician-administered, interviewbased instrument, making it unsuitable for use in community settings; moreover, its self-report format is too lengthy. Other measures such as the Obsessive Compulsive Inventory - Child Version (OCI-CV), ${ }^{11}$ the Children's Obsessional Compulsive Inventory (CHOCI) ${ }^{12}$ the Children's Florida Obsessive-Compulsive Inventory (C-FOCI) ${ }^{13}$ the Obsessive-Compulsive Subscale (OCS) of the Child Behavior Checklist (CBCL) ${ }^{14}$ and the Child Saving Inventory $(\mathrm{CSI})^{15}$ are promising self-report measures; however, all of them present some limitations. These limitations concern features such as the tools' length, ease of use and availability. ${ }^{16}$ Furthermore, most researchers assert that evidence-based assessment (EBA) is a first step towards the development of psychopathological studies and assessment of the effectiveness of empirically validated treatments. ${ }^{9,16}$ Specifically, the NICE recommends the Short Obsessive-Compulsive Disorder Screener (SOCS). ${ }^{6,7}$

Uher $e \mathrm{al}^{7}$ designed and validated the SOCS to provide a simple screening tool suitable for general practice and community settings. The SOCS is a seven-item self-report tool with a three-point response format ( 0 ['no'], 1 ['a bit'] and 2 ['a lot']). The first five items were adapted from the five most discriminant items of the 44-item child version of the Leyton Obsessional Inventory (LOI) ${ }^{17}$ and assess common symptoms including checking, touching, cleanliness/washing, repeating and exactness. Two additional questions assess the associated impairment and resistance. The SOCS score is calculated by summing the scores for all seven items. With a sample of 11- to 15-year-old youth, Uher et al found that the tool had good internal consistency, a unidimensional factor structure and excellent sensitivity for detecting OCD in children; furthermore, its specificity was good for children without psychiatric diagnoses but poor for a psychiatric sample. Although Uher et al's study highlights the tool's uses, the SOCS still has limitations concerning certain psychometric properties, such as factorial structure invariance, test-retest reliability, and the lack of evidence of convergent/ divergent validity. Furthermore, all psychometric properties must be investigated in a broader age range than 11-15 years. 
Consequently, our main objective was to further validate the SOCS while empirically examining whether it is useful in assessing obsessive-compulsive symptoms across a wide age range of clinical and community samples of children and adolescents.

\section{Method}

\section{Participants}

The clinical sample comprised 94 consecutive child and adolescent patients (46 males; 48 females) with a current diagnosis of OCD according to the DSM-IV criteria. ${ }^{18}$ Their mean age was 14.62 years (s.d.=2.65; range 9-19). All patients were recruited from the Child and Adolescent Psychiatry and Psychology Unit of Hospital Clínic de Barcelona, and from the Child and Adolescent Psychological Clinical Unit of Universidad Miguel Hernández de Elche. Axis I diagnoses were ascertained using the Schedule for Affective Disorders and Schizophrenia for School-Age Children Present and Lifetime Version (K-SADS-PL), ${ }^{19}$ which has been validated in Spanish. ${ }^{20}$ The children and their parents were informants. Exclusion criteria were psychiatric comorbidity with a psychotic disorder or autism spectrum disorder, and an IQ of less than 70. Patients could have another comorbid Axis I disorder, but OCD needed to be the primary disorder. Specifically, we found the following rates of associated secondary disorders: generalised anxiety disorder $16 \%$, Tourette's disorder $4.3 \%$, attention-deficit hyperactivity disorder $4.3 \%$, social phobia $2.1 \%$ and major depression $2.1 \%$.

Written informed consent was obtained from all participants and families following an explanation of the procedures involved.

The community sample consisted of 880 students ( 437 males; 443 females) from five schools in Levante, eastern Spain. Their mean age was 13.21 years (s.d.=2.19; range 10-18). All the schools volunteered for the study. After obtaining permission from the principals and psycho-pedagogical departments of the schools, all students agreed to participate.

\section{Measures}

SOCS $^{7}$

This was translated into Spanish with translation and backtranslation. ${ }^{21}$ The Spanish version of the SOCS was administered to a pilot sample of 10-year-olds to ensure that the item content could be understood by young children. This Spanish translation was authorised in 2009 by Isobel Heyman, an author of the original scale.

\section{Ad hoc sociodemographic sheet}

This was designed to collect data on age, gender and geographic area of residence.

\section{Spanish version of the OCl-CV}

This comprises 21 items scored on a 3-point Likert scale (0 ['never'], 1 ['sometimes'] and 2 ['always']). The OCI-CV provides six subscale scores - doubting/checking, obsessing, hoarding, washing, ordering and neutralising - and a total score. ${ }^{11}$ The psychometric properties of the English ${ }^{11}$ and Spanish ${ }^{16}$ versions have been reported.

\section{C-FOCl ${ }^{13}$}

This was developed from the original version of the FOCI for adults to assess obsessive-compulsive symptoms in 7- to 20-yearold children and adolescents. It consists of 22 items in two subscales: symptom checklist and OCD severity scale. It has shown adequate psychometric properties both for English ${ }^{13}$ and
Spanish speaking children and adolescents (further details of the Spanish validation study available from the authors on request).

\section{Revised Child Anxiety and Depression Scale (RCADS-30)22}

This is a reduced 30 -item version of the RCADS, ${ }^{23}$ a self-report for evaluating anxiety and depression in children and adolescents. The scale comprises 30 items and in six subscales for evaluating symptoms of the following conditions: panic disorder, social phobia, separation anxiety disorder, generalised anxiety disorder, OCD and major depressive disorder. The scale ranges from 0 to 3 (corresponding to never, sometimes, often and always, respectively). The scale has shown excellent psychometric properties, equivalent to the full version, with a Spanish population. ${ }^{22}$

\section{CY-BOCS}

This is a clinician-administered scale involving a semi-structured interview for assessing the severity of obsessive-compulsive symptoms in children. ${ }^{10}$ The scale includes a list of subdomains used to identify the content of the patient's obsessions and compulsions. These symptoms are then rated on a 5-point scale (0 ['no symptoms'] to 4 ['extreme symptoms']) covering five areas: time spent on the symptoms per day, interference caused by symptoms, level of distress, level of resistance and control. Summing these scores for each symptom produces scores for the severity of various obsessions and compulsions. A total severity score (ranging from 0 to 40) can also be obtained, with a higher score indicating greater severity. A total severity score of 16 serves as the cut-off point for an OCD diagnosis.

\section{Procedure}

The study was approved by the Research and Ethics Committees of the Universidad Miguel Hernández de Elche and the Hospital Clínic de Barcelona. Experienced psychologists with master's degree or doctorate in psychology administered the SOCS and the other self-administered questionnaires to the clinical and community samples at the time of study entry, along with the $\mathrm{K}-\mathrm{SADS}-\mathrm{PL}$ and CY-BOCS exclusively to the clinical sample. They administered the questionnaires 4 weeks after the first evaluation again. The psychologists gave the participants clear instructions concerning how to indicate their responses and they were reassured that it was not a test and that there were no right or wrong answers, and individual assistance to those students who needed it. In order to prevent the introduction of biases in the collection of information, four different balanced protocols were applied. For it, the questionnaires were randomly ordered in each protocol. The average time of application of the assessment was about 50 minutes and no cases of fatigue were detected.

\section{Data analysis}

Six sets of analyses based on the ordinal nature of the variables (in all cases, we used ordinal and not ratio scales) were conducted.

First, we examined the item distribution and frequency as well as the factor structure of the SOCS for the clinical and community samples. Then, based on the results of Uher et al, ${ }^{7}$ the hypothesis that the SOCS has a single-factor structure was tested by confirmatory factor analysis (CFA) performed simultaneously for both the clinical and community samples. We also tested the alternative hypothesis that the SOCS items are independent, and tested whether the SOCS exhibits metric invariance. Testing metric invariance was conducted once, not systematically (i.e. we did not check the configuration invariance first; instead, we first checked the invariance of the factor loadings, after which we checked the measurement errors followed by the intercepts). Therefore, in all cases, we introduced all the restrictions 


\begin{tabular}{|c|c|c|c|c|c|c|c|c|}
\hline Sample/model & $\chi^{2}$ & $\mathrm{df}$ & RMSEA ( $90 \% \mathrm{Cl})$ & $\mathrm{CFI}$ & GFI & NNFI & $\mathrm{IFI}$ & RFI \\
\hline \multicolumn{9}{|l|}{ Total sample } \\
\hline Independent & 1969.59 & 21 & & & & & & \\
\hline One factor & 55.55 & 14 & $0.06(0.04-0.07)$ & 0.98 & 0.99 & 0.97 & 0.98 & 0.96 \\
\hline \multicolumn{9}{|c|}{ Metric invariance } \\
\hline \multicolumn{9}{|c|}{ Clinical community } \\
\hline Independent & 1779.37 & 42 & & & & & & \\
\hline One factor & 109.59 & 55 & $0.05(0.03-0.06)$ & 0.97 & $\begin{array}{c}\text { Clinical: } 0.90 \\
\text { Community: } 0.99\end{array}$ & 0.98 & 0.97 & 0.95 \\
\hline
\end{tabular}

simultaneously, which enabled us to check the metric invariance between the samples with a single calculation. We used polychoric correlation matrices and the diagonally weighted least squares (DWLS) method in all cases (LISREL 8.8, DWLS procedure). Last, we used the following indices as goodness-of-fit measures ${ }^{24}$ : the Satorra-Bentler chi-square, a root mean square error of approximation (RMSEA) less than or equal to 0.8 and a confirmatory factor index (CFI), goodness of fit index (GFI) and non-normed fit index (NNFI) (or Tucker-Lewis index) higher than 0.95. The CFI was also used to compare the basic model (total sample) with the metric invariance model in the case of the two samples. According to Cheung and Rensvold, ${ }^{25}$ the invariance between samples is admissible when the difference between the CFIs $(\Delta \mathrm{CFI})$ is less than or equal to 0.01 . They also assert that $\Delta \mathrm{CFI}$ is a better estimator of invariance admissibility than $\Delta \chi^{2}$, which is the index used by some researchers.

McDonald's omega ${ }^{26}$ and the greatest lower bound to reliability index $(\mathrm{GLB})^{27}$ were used to estimate the internal consistency of the SOCS, as they are better estimators of reliability than the Cronbach's alpha is. ${ }^{28}$ We also administered the SOCS twice after an interval of 4 weeks to examine test-retest reliability.

Discriminative validity was examined using the MannWhitney $U$-test and the probability of superiority ${ }^{29}$ was used to estimate the effect size of the differences between the samples.

We also conducted analyses of convergent/divergent validity by calculating Spearman's rho coefficients. We used Cohen's criteria $^{30}$ to evaluate the effect sizes of the correlations: small $(0.20)$ and large $(\geq 0.50) .^{30,31}$

To determine the accuracy of the scales in terms of their ability to correctly classify children and adolescents with or without $\mathrm{OCD}$, we conducted a receiver operating characteristic (ROC) curve analysis, wherein subscales with areas under the curve (AUCs) between 0.70 and 0.80 were considered adequate.

Then, the predictive validity of the SOCS for a K-SADS-PL diagnosis of OCD was calculated by binary logistic regression analyses. The diagnoses according to the K-SADS-PL were the outcome variables (OCD or non-OCD), and the predictor variable was SOCS score.

\section{Results}

\section{Item analysis}

The means of the item scores for the clinical sample ranged from 0.66 (item 4) to 1.31 (item 1), and the standard deviations ranged from 0.59 (item 7) to 0.80 (item 2). The distribution for almost all the item scores showed skewness indices $(-0.57$ to 0.70$)$ and kurtosis indices $(-1.33$ to 0.46$)$ near zero. Overall, the average percentage of each response across the items scores was as follows: 'no' (26\%), 'a lot' (34.1\%) and 'a bit' (39.9\%). In the community sample, the means of the items scores ranged from 0.55 (item 6) to 1.05 (item 5), and the standard deviations ranged from 0.63 (item 1) to 0.75 (item 3). For almost all the items scores, skewness $(-0.09$ to 0.80$)$ and kurtosis indices $(-1.20$ to 0.46$)$ were near zero. Overall, the average percentage of each response across the items scores was as follows: 'a lot' (18.4\%), 'no' (37.8\%) and 'a bit' (43.8\%).

\section{CFA}

As can be seen in Table 1, for the total sample (clinical and community), the fit indices indicated that the model fits the data reasonably well, and we found metric invariance between the samples (configurational invariance and invariance of factor loadings, measurement errors and intercepts). The RMSEA was $<0.8$, and the CFI, GFI and NNFI were $>0.90$; the $\Delta$ CFI was 0.01 (0.98-0.97).

Table 2 shows the degree of the relationship (standardised lambda weights) for each item score with a single factor. As is evident, all item weights are above 0.50 , except for the weight of item 2 (0.35).

\section{Reliability}

The internal consistency (McDonald's omega and GLB) of the SOCS for both samples is provided in Table 3. The reliability indices for the total score were higher in the clinical sample.

Regarding test-retest reliability, the two mean SOCS scores of the clinical sample were 7.56 (s.d.=3.15) and 6.58 (s.d.=3.18), with a rho of 0.79 . In the community sample, the mean scores were 5.64 (s.d.=2.83) and 4.61 (s.d.=2.83), with a rho of 0.63 . In both samples, the correlations were statistically significant $(P<0.01)$.

\begin{tabular}{|c|c|c|}
\hline Item & Item content & Lambda \\
\hline 1 & $\begin{array}{l}\text { Does your mind often make you do things like checking } \\
\text { or touching things or counting things, even though you } \\
\text { know you don't really have to? }\end{array}$ & 0.50 \\
\hline 2 & Are you particularly fussy about keeping your hands clean? & 0.35 \\
\hline 3 & $\begin{array}{l}\text { Do you ever have to do things over and over a certain } \\
\text { number of times before they seem quite right? }\end{array}$ & 0.61 \\
\hline 4 & $\begin{array}{l}\text { Do you ever have trouble finishing your school work or } \\
\text { chores because you have to do something over and } \\
\text { over again? }\end{array}$ & 0.56 \\
\hline 5 & $\begin{array}{l}\text { Do you worry a lot if you've done something not exactly } \\
\text { the way you like? }\end{array}$ & 0.54 \\
\hline 6 & Do these things interfere with your life? & 0.78 \\
\hline 7 & Do you try to stop them? & 0.51 \\
\hline
\end{tabular}


Table 3 Means (s.d.), differences between clinical and community samples and reliability (MCDonald's omega and GLB) fo the socs. Total sample $(N=974)$, children from clinical setting $(n=94)$ and children from the community $(n=880)$.

\begin{tabular}{llc|}
\hline Sample & Statistic & Total score \\
\hline Total sample & Omega & 0.75 \\
& GLB & 0.80 \\
& Mean & 5.82 \\
& S.d. & 2.92 \\
\hline Clinical sample & Omega & 0.79 \\
& GLB & 0.88 \\
& Mean & 7.56 \\
Community sample & S.d. & 3.15 \\
& Omega & 0.74 \\
& GLB & 0.79 \\
& Mean & 5.64 \\
Mann-Whitney U-test & S.d. & 2.83 \\
$Z$ & & $27093^{*}$ \\
PS effect size & -5.53 \\
GLB, greatest lower bound to reliability; PS, probability that a randomly sampled score \\
from one population is larger than a randomly sampled score from a second \\
population. & \\
${ }^{*} P=0.01$. & \\
\hline
\end{tabular}

\section{Descriptive statistics of the scales and discriminative validity}

Table 3 shows the means and standard deviations of the SOCS for the total sample, for the clinical sample and for the community sample. In general, the students from the clinical sample obtained higher scores on the SOCS than did those from the school sample (probability of superiority 0.67 , which is considered a medium effect size according to Grissom ${ }^{33}$ ).

\section{Analysis of convergent/divergent validity}

The results indicated that in both samples, SOCS score was significantly and positively correlated with the scores on wellestablished measures of child OCD (see Table 4). The effect sizes were large in the clinical sample $(r=0.50-0.80)$ and medium to large in the community sample $(r=0.47-0.72)$. The only exception, in both samples, was the OCI-CV's Hoarding scale, which had a moderate correlation with the SOCS. In the clinical sample, the total severity scale of the CY-BOCS showed a moderate association with the SOCS.
Table 4 Convergent/divergent validity of the Socs. Total

sample $(N=974)$, children from clinical setting $(n=94)$ and children from the community $(n=880)$.

\begin{tabular}{|c|c|c|}
\hline \multirow[t]{2}{*}{ Measures } & \multicolumn{2}{|c|}{ sOCS } \\
\hline & Clinical & Community \\
\hline CY-BOCS total severity score & $0.38^{* *}$ & - \\
\hline C-FOCl symptom checklist & $0.65^{\star *}$ & $0.61^{\star *}$ \\
\hline C-FOCI severity scale & $0.57^{* *}$ & $0.49^{* *}$ \\
\hline OCl-CV checking & $0.68^{\star \star}$ & $0.62^{\star \star}$ \\
\hline OCl-CV obsessing & $0.53^{* *}$ & $0.47^{* *}$ \\
\hline OCl-CV hoarding & $0.43^{\star \star}$ & $0.43^{\star *}$ \\
\hline OCl-CV washing & $0.53^{\star \star}$ & $0.54^{\star *}$ \\
\hline OCl-CV ordering & $0.50^{\star *}$ & $0.49^{* *}$ \\
\hline OCl-CV neutralising & $0.58^{\star *}$ & $0.47^{* *}$ \\
\hline OCl-CV total score & $0.80^{\star *}$ & $0.72^{\star *}$ \\
\hline RCADS-30 separation anxiety & $0.26^{*}$ & $0.36^{* *}$ \\
\hline RCADS-30 social phobia & $0.43^{\star *}$ & $0.40^{\star *}$ \\
\hline RCADS-30 generalised anxiety & $0.46^{\star *}$ & $0.43^{\star *}$ \\
\hline RCADS-30 panic disorder & $0.44^{\star \star}$ & $0.42^{\star *}$ \\
\hline RCADS-30 obsessive-compulsive disorder & $0.63^{\star \star}$ & $0.52^{\star \star}$ \\
\hline RCADS-30 major depression & $0.49^{* *}$ & $0.41^{* *}$ \\
\hline RCADS-30 total score & $0.61^{* *}$ & $0.57^{* *}$ \\
\hline \multicolumn{3}{|c|}{$\begin{array}{l}\text { SOCS, Short OCD Screener; OCI-CV, Obsessive-Compulsive Inventory - Child Version; } \\
\text { RCADS- } 30 \text {, Revised Child Anxiety and Depression Scale; C-FOCI, Children’s Florida } \\
\text { Obsessive-Compulsive Inventory; CY-BOCS, Children's Yale-Brown Obsessive Com- } \\
\text { pulsive Scale. } \\
\star P<0.05 ;{ }^{*} P<0.01 \text {. }\end{array}$} \\
\hline
\end{tabular}

With regard to related construct measures such as anxiety and depression symptoms, all the correlations were significant with medium effect sizes $(r=0.26-0.49)$. The only exception in both samples was the total score on the RCADS, which showed a large effect size (see Table 4).

\section{Sensitivity and specificity of the SOCS}

The AUC of the SOCS was 0.67 (s.e. $=0.03, P<0.01$ ), suggesting that there is a $67 \%$ probability that a child or adolescent with OCD will have a higher score on the SOCS than a non-OCD child/ adolescent. Table 5 shows the sensitivity and specificity of the SOCS.

We selected cut-off scores to provide the best balance between sensitivity and specificity for each measure. A SOCS cut-off score of 7 showed a sensitivity of $61 \%$ and a specificity of $60 \%$, a Youden index of 0.21 and an accuracy or informedness (percentage of participants correctly classified) of $40 \%$. However, according to the Youden index and accuracy percentage, a score of

Table 5 Sensitivity, specificity, Youden index and accuracy of the socs. Total sample $(N=974)$, children from clinical setting $(n=94)$ and

\begin{tabular}{|c|c|c|c|c|c|c|}
\hline Cut-off & Sensitivity & Specificity & PPV & NPV & Youden index & Accuracy \\
\hline 1 & 0.98 & 0.02 & 0.11 & 0.90 & 0.00 & 0.89 \\
\hline 2 & 0.97 & 0.08 & 0.04 & 0.90 & 0.05 & 0.84 \\
\hline 3 & 0.95 & 0.15 & 0.04 & 0.89 & 0.09 & 0.78 \\
\hline 4 & 0.90 & 0.26 & 0.04 & 0.89 & 0.16 & 0.68 \\
\hline 5 & 0.83 & 0.37 & 0.05 & 0.88 & 0.20 & 0.59 \\
\hline 6 & 0.76 & 0.49 & 0.05 & 0.86 & 0.25 & 0.49 \\
\hline 7 & 0.61 & 0.60 & 0.07 & 0.86 & 0.21 & 0.40 \\
\hline 8 & 0.51 & 0.73 & 0.07 & 0.83 & 0.24 & 0.29 \\
\hline 9 & 0.37 & 0.82 & 0.08 & 0.82 & 0.19 & 0.22 \\
\hline 10 & 0.30 & 0.91 & 0.08 & 0.74 & 0.21 & 0.15 \\
\hline 11 & 0.21 & 0.96 & 0.08 & 0.62 & 0.18 & 0.11 \\
\hline 12 & 0.12 & 0.98 & 0.09 & 0.56 & 0.10 & 0.10 \\
\hline 13 & 0.04 & 1.00 & 0.09 & 0.50 & 0.04 & 0.10 \\
\hline 14 & 0.02 & 1.00 & 0.09 & 0.00 & 0.02 & 0.09 \\
\hline
\end{tabular}


6 was more sensitive but less specific, making it a possibly more suitable cut-off score for detecting OCD.

\section{Predictive validity}

The predictive model accounted for $8 \%$ (Nagelkerke's $R^{2}$ ) of the variance in OCD diagnoses and resulted in a classification accuracy of $90.3 \%$. The SOCS was related to diagnostic status (Wald $(1)=34.66, P<0.01)$ such that higher SOCS scores were better predictors of OCD diagnoses. The odds ratio was significant, OR=1.26 (95\% CI 1.17-1.36).

\section{Discussion}

The NICE recommends the SOCS as a suitable and validated screening tool for youth between 11 and 15 years. In spite of its excellent sensitivity and specificity in detecting OCD in nonclinical children, the research on SOCS does show some gaps. For example, the invariance of the SOCS' factorial structure is unknown, there is some ambiguity regarding test-retest reliability estimations and there is a lack of evidence concerning its convergent/divergent validity. Moreover, all such properties must be determined for a broader age range than 11- to 15-year-olds. Thus, we provided new data about these psychometric properties to determine whether the SOCS is suitable for assessing OCD symptoms across a wider age range of children and adolescents.

Our results supported the factorial validity of the SOCS, confirming the unidimensional structure previously found. ${ }^{7}$ The CFA showed that the data fitted adequately to the unidimensional measurement model, although the factor loadings of item 2 were below 0.40 . This small load factor for item 2 may be due to hypothetical understanding problem by the youngest children.

With regard to invariance, the SOCS presented the expected metric invariance between the samples, as indicated by an RMSEA below 0.06 and CFI, GFI and NNFI values over 0.90; these values also indicated acceptable fit according to Schermelleh-Engel et $a l^{24}$ Thus, in both samples, we found the same factor and equivalent factor loadings, measurement errors and intercepts.

With respect to reliability estimation, the internal consistency coefficients of the SOCS were between moderate and high (0.74-0.88), namely, above the recommended value of 0.70 proposed by Nunnally \& Bernstein. ${ }^{33}$ These data indicate that the scale has a high internal consistency, which supports the existence of a single dimension. The data are consistent with a previous study carried out with the SOCS, which also indicated good internal consistency (Cronbach's alpha 0.85). Our data are also consistent with those concerning other EBA measures for OCD, such as the CY-BOCS, OCI-CV, CHOCI, C-FOCI, OCS and CSI. ${ }^{9}$

The SOCS also showed an adequate temporal stability, as we found positive correlations for both the clinical (0.79) and community (0.63) samples after 4 weeks. According to reliability studies, values between 0.50 and 0.70 indicate moderate reliability, and values between 0.80 and 0.90 indicate good reliability. Other studies on self-report measures for OCD show similar short-term temporal stability. ${ }^{9}$

The mean score of the clinical sample was higher than that of the community sample $(M=5.64$; s.d. $=2.83)$, with a medium effect size, supporting the discriminative validity of the SOCS. In addition, the mean score for the OCD group was lower than that reported by Uher et $a l^{7}$ for an OCD group $(M=9.7$, s.d.=2.2) but higher than the mean for Uher et al's healthy group $(M=3$, s.d.=2.3), mixed community group $(M=3.3$, s.d.=2.5) and psychiatric control group $(M=5.8$, s.d.=2.8). Compared with the results of Uher et al, ${ }^{7}$ our results revealed somewhat low levels of obsessivecompulsive symptoms in the clinical sample but high levels in the community sample. This result could be because we obtained SOCS scores from samples with mean ages and an age range higher than was the case in Uher et $a l^{7}$ and from a community sample larger than that in Uher et al..$^{7}$ Furthermore, we calculated the mean of the community sample without eliminating the scores of children who could have met the criteria for OCD (because we did not evaluate these criteria in this sample), whereas Uher et al eliminated children who met OCD criteria from their community sample. Therefore, in our sample, children with potential OCD were also considered when calculating the mean SOCS score. However, our outcomes are similar to findings concerning other OCD screening instruments (i.e. the LOI-CV), which displayed sensitivity values between poor and adequate. ${ }^{9}$ This conclusion can be defended as SOCS scores have metric invariance and the same intercepts in clinical and nonclinical populations, an issue supported in our study.

Regarding the concurrent validity, the confirmed relationships between the SOCS and the three measures with the highest empirical support - the CY-BOCS, OCI-CV and C-FOCI - as indicated by Iniesta-Sepúlveda et $a l,{ }^{9}$ also supports the validity of the SOCS. A large positive relationship was found between the SOCS and the total scores on the OCI-CV and the severity scale of the C-FOCI, whereas a moderate association was found between the SOCS and the total severity scale of the CY-BOCS in the clinical sample, following Cohen's effect size criteria. ${ }^{30,31}$ However, there were also moderate associations between the SOCS and specific anxiety and depression symptom measures. One explanation for this finding is the symptom overlap between depression, anxiety and OCD disorders, which other studies have also found. ${ }^{34}$

Finally, we examined the sensitivity and specificity of the SOCS by using ROC analysis. Overall, the results indicated an AUC of 0.67 . Values around 0.70 are considered to represent acceptable discriminatory ability. According to our data, a SOCS score above 7 was initially recommended because it provides an optimal balance between the percentages of true positives and negatives in real cases. This cut-off score led to an acceptably low percentage of adolescents incorrectly identified as having OCD (specificity 60\%) and an adequate proportion of adolescents being overlooked in terms of heightened OCD symptoms (sensitivity $61 \%$ ). However, because the main purpose of the SOCS is to serve as a screening measure of $\mathrm{OCD}$, the best cut-off score is 6 , with a Youden index of 0.25 . This SOCS score could be advantageous for screening adolescents in clinical settings, as it would preclude the overlooking of adolescents requiring further assessment. However, depending on the intended purpose of the SOCS, administrators could choose a higher or lower cut-off score. For example, a more conservative cut-off score of $8-9$ might be justified when using the subscales of the SOCS for research purposes in order to avoid false positives (see Table 5). Our sensitivity/specificity results were lower than those reported by Uher et $a l^{7}$ but again, the sample differences could explain this discrepancy. However, our outcomes are similar to findings concerning other OCD screening instruments (i.e. the LOI-CV), which displayed sensitivity values between poor and adequate. ${ }^{9}$ However, this aspect of OCD measures presents limited psychometric evidence according to Iniesta-Sepúlveda et al. ${ }^{9}$ Furthermore, although the community sample was assumed to have no OCD cases, it is possible that this sample contained such cases, which could explain our relatively low sensitivity and specificity.

Regarding the predictive validity of the SOCS, the results of a binary logistic regression analysis indicated that the scale was a significant predictor of OCD diagnosis (classification accuracy of 90.3\%). This result is consistent with the findings of Uher et al's study with the SOCS, ${ }^{7}$ which resulted in the development of a screening tool suitable for accurately classifying OCD. 


\section{Limitations}

Some limitations should be noted. First, our study did not examine the psychometric properties of SOCS with a large clinical sample. Second, the sensitivity and specificity in differentiating children with and without OCD with a sample of children with the disorder and a non-clinically interviewed community-based sample. And third, its convergent and discriminant validity with other self-report measures as well as the relationship of its scores with those from other assessment procedures (e.g. information deriving from parents or teachers).

\section{Use of the SOcS}

In conclusion, the SOCS is shorter than other self-report tools (for further details, see the review by Iniesta-Sepúlveda et $a l^{9}$ ) and has comparable psychometric properties. Following the classification of EBAs, ${ }^{35}$ which are based on three levels of empirical support, and according to the results of this and Uher et al's study, ${ }^{7}$ the SOCS can be considered a well-established, or at least a promising, paediatric OCD assessment instrument.

\section{Acknowledgements}

We thank Isobel Heyman for permitting us to use the SoCS, David Mataix-Cols and Miquel A Fullana for their assistance in translating the tool into Spanish and Agustin E. MartínezGonzález for his drive and dedication.

José A. Piqueras, PhD, Tíscar Rodríguez-Jiménez, Universidad Miguel Hernández de Elche, Alicante, Spain; Ana G. Ortiz, ScM, Elena Moreno, ScM, Department of Child and Adolescent Psychiatry and Psychology, Hospital Clínic de Barcelona, Barcelona, Spain; Luisa Lázaro, PhD, Department of Child and Adolescent Psychiatry and Psychology, Hospital Clínic de Barcelona, IDIBAPS. CIBERSAM, University of Barcelona, Barcelona, Spain; Antonio Godoy, PhD, Universidad de Málaga, Málaga, Spain

Correspondence: José A. Piqueras, Universidad Miguel Hernández de Elche Avda. de la Universidad, s/n 03202 Elche (Alicante, Spain). E-mail: jpiqueras@umh.es First received 19 Apr 2015, final revision 2 Jun 2015, accepted 4 Jun 2015

\section{References}

1 American Psychiatric Association. Diagnostic and Statistical Manual of Mental Disorders (5th edn). APA, 2013.

2 Farrell L, Barrett PM. Obsessive-compulsive disorder across developmenta trajectory: cognitive processing of threat in children, adolescents and adults. Br J Psychol 2006; 97: 95-114.

3 Canals J, Hernández-Martínez C, Cosi S, Voltas N. The epidemiology of obsessivecompulsive disorder in Spanish school children. J Anxiety Disord 2012; 26: 746-52.

4 Godoy A, Gavino A, Carrillo F, Cobos MP, Quintero C. Composición factorial de la versión española de la Spence Children Anxiety Scale (SCAS). Psicothema 2011; 23: 289-94.

5 Orgilés M, Méndez X, Espada JP, Carballo JL, Piqueras JA. Anxiety disorde symptoms in children and adolescents: differences by age and gender in community sample. Rev Psiquiatr y Salud Ment (English Ed) 2012; 5: 115-20.

6 Krebs G, Heyman I. Obsessive-compulsive disorder in children and adolescents. Arch Dis Child 2014; 100: 495-9.

7 Uher R, Heyman I, Mortimore C, Frampton I, Goodman R. Screening young people for obsessive-compulsive disorder. Br J Psychiatry 2007; 191: 353-4.

8 Overduin MK, Furnham A. Assessing obsessive-compulsive disorder (OCD): a review of self-report measures. J Obsessive Compuls Relat Disord 2012; 1: 1-13.

9 Iniesta-Sepúlveda M, Rosa-Alcázar Al, Rosa-Alcázar Á, Storch EA. Evidence-based assessment in children and adolescents with obsessive-compulsive disorder. Child Fam Stud 2013; 23: 1455-70.

10 Scahill L, Riddle MA, McSwiggin-Hardin M, Ort SI, King RA, Goodman WK, et al. Children's Yale-Brown Obsessive Compulsive Scale: reliability and validity. J Am Acad Child Adolesc Psychiatry 1997: 36: 844-52.
11 Foa EB, Coles ME, Huppert JD, Pasupuleti RV, Franklin ME, March JS. Development and validation of a child version of the obsessive compulsive inventory. Behav Ther 2010; 41: 121-32.

12 Shafran R, Frampton I, Heyman I, Reynolds M, Teachman B, Rachman S. The preliminary development of a new self-report measure for OCD in young people. J Adolesc 2003; 26: 137-42.

13 Storch EA, Khanna M, Merlo ப, Loew BA, Franklin M, Reid JM. Children's Florida obsessive compulsive inventory: psychometric properties and feasibility of a selfreport measure of obsessive-compulsive symptoms in youth. Child Psychiatry Hum Dev 2009: 40: 467-83.

14 Achenbach TM. Manual for the Child Behavior Checklist/4-18 and 1991 Profile. University of Vermont Department of Psychiatry, 1991

15 Storch EA, Muroff J, Lewin AB, Geller D, Ross A, McCarthy K, et al. Development and preliminary psychometric evaluation of the children's saving inventory. Child Psychiatry Hum Dev 2011; 42: 166-82.

16 Rodríguez-Jiménez T, Godoy A, Piqueras JA, Gavino A Martínez-González AE, Foa E. Factor structure and measurement invariance of the Obsessive-Compulsive Inventory - Child version (OCl-CV) in general population. Eur J Psychol Assess 2015, in press (doi: 10.1027/1015-5759/a000276).

17 Berg CJ, Rapoport JL, Flament M. The leyton obsessional inventory-child version. J Am Acad Child Psychiatry 1986; 25: 84-91.

18 Association AP. Diagnostic and Statistical Manual of Mental Disorder (DSM-IV) (4th edn). American Psychiatric Association, 1994.

19 Kaufman J, Birmaher B, Brent D, Rao U, Flynn C, Moreci P, et al. Schedule for affective disorders and schizophrenia for school-age children-present and lifetime version (K-SADS-PL): initial reliability and validity data. J Am Acad Child Adolesc Psychiatry 1997; 36: 980-8.

20 Ulloa RE, Ortiz S, Higuera F, Nogales I, Fresán A, Apiquian $R$, et al Interrater reliability of the Spanish version of schedule for affective disorders and schizophrenia for school-age children-present and lifetime version (K-SADS-PL). Actas Esp Psiquiatr 2006; 34: 36-40.

21 Hambleton RK, Merenda P, Spielberger C (eds). Adapting Educational and Psychological Tests for Cross-cultural Assessment. Lawrence Erlbaum, 2005.

22 Sandin B, Chorot P, Valiente RM, Chorpita BF, Sandín B. Development of a 30-item version of the revised child anxiety and depression scale. Rev Psicopatología y Psicol Clínica 2010; 15: 165-78

23 Chorpita BF, Yim L, Moffitt CE, Umemoto LA, Francis SE. Assessment of symptoms of DSM-IV anxiety and depression in children: a revised child anxiety and depression scale. Behav Res Ther 2000; 38: 835-55.

24 Schermelleh-Engel $\mathrm{K}$, Moosbrugger $\mathrm{H}$, Müller $\mathrm{H}$. Evaluating the fit of structural equation models: tests of significance and descriptive goodness-of-fit measures. Methods Psychol Res Online 2003; 8: 23-74.

25 Cheung GW, Rensvold RB. Evaluating goodness-of-fit indexes for testing measurement invariance. Struct Equ Model A Multidiscip J 2002; 9: 233-55.

26 McDonald R. Test Theory: A Unified Treatment. Lawrence Erlbaum, 1999.

27 Woodhouse B, Jackson PH. Lower bounds for the reliability of the total score on a test composed of non-homogeneous items: $\|$ : a search procedure to locate the greatest lower bound. Psychometrika 1977; 42: 579-91.

28 Schweizer $\mathrm{K}$. On the changing role of Cronbach's $\alpha$ in the evaluation of the quality of a measure. Eur J Psychol Assess 2011; 27: 143-4.

29 Erceg-Hurn DM, Mirosevich VM. Modern robust statistical methods: an easy way to maximize the accuracy and power of your research. Am Psychol 2008; 63: $591-601$.

30 Cohen J. Statistical Power Analysis for the Behavioral Science (2nd edn). Lawrence Erlbaum, 1988

31 Lipsey, M, Wilson D. Practical Meta-analysis. Sage, 2001.

32 Grissom RJ. Probability of the superior outcome of one treatment over another J Appl Psychol 1994; 79: 314-16.

33 Nunnally JC, Bernstein IH. Psychometric Theory. McGraw-Hill, 1994.

34 Sun J, Li Z, Buys N, Storch EA. Correlates of comorbid depression, anxiety and helplessness with obsessive-compulsive disorder in Chinese adolescents. J Affect Disord 2015; 174: 31-7.

35 Cohen LL, Cohen LL, La Greca AM, La Greca AM, Blount RL, Blount RL, et al. 2007 . Introduction to special issue: evidence-based assessment in pediatric psychology. J Pediatr Psychol 2008; 33: 911-5. 